\title{
Modélisation de la résistance aux chocs de salinité des postlarves de Penaeus vannamei
}

\author{
AQUACOP, Gilles Le Moullac et Didier Damez \\ IFREMER, BP ך(No, Taravan, Tahiti, Polynésie Française. \\ Reçu le 30 janvier Iggr; accepté le 6 mai $19 g 1$.
}

Modeling of resistance to salinity shocks of Penaeus tannamei postlarvae.

AQUACOP, G. Le Moullac, D. Damez. Aquat. Lizing Resour., 1991, 4, 169-173.

\begin{abstract}
The notion of penaeid shrimp postlarvae quality, which implics a survival potential, is still a subjective parameter. The zootechnical criteria and the visual observations are not sufficient to cvaluate the physiological condition of the hatchery reared postlarvae. The resistance of postlarvae to salinity shocks is a representative criteria of their condition. The goal of this study is to present a non-linear regression model at 2 variables (size, salinity) for the $P$. vannamei postlarvac resistance to salinity shocks. Between the second and the twenticth day after metamorphosis; 5 salinity shocks $(20,15,10$, 5 and $2 \%$ ) were tested daily, each one with 50 postlarvac. Survival rates were recorded after $2 \mathrm{hrs}$. Concurrently, the postlarvae growth in the rearing tank was studicd by the daily measurement of 30 individuals. The survival to the salinity shocks improves with the size which increases linearly.
\end{abstract}

Keywords : Penaeus tannamei, postlarvae, growth, resistance, salinity.

Résumé

La notion de qualité des postlarves de crevettes pénéides, qui sous-entend un potentiel de survie, est un paramètre resté longtemps subjectif. Les critères zootechniques et les observations visuelles ne sont pas des paramètres suffisants pour apprécier la condition physiologique des postlarves à la sortie de l'écloserie. La résistance aux chocs de salinité est un critèrc représentatil de l'état des postlarves. Cette étude a pour but de présenter un modèle de régression non linéaire à deux variables (taille, salinité) de la résistance des postlarves Penacus vannamei à des chocs de salinité. Entre le $2^{\mathrm{e}}$ et le $20^{\mathrm{e}}$ jour après la métamorphose 5 chocs de salinité $(20,15,10,5$ et $2 \%$ ) sont testés chaque jour, avec respectivement 50 postlarves par salinité. Le taux de survic est enregistré après 2 heures. Parallèlement, la croissance des postlarves en ćlcvage est étudiée par la mesure quotidienne de 30 animaux. La tolérance à la salinité des postlarves $P$. vannamei augmente avec la taille qui s'accroît d'une façon linéaire.

Mots-clés : Penaeus vannamei, postlarve, croissance, résistance, salinité.

\section{INTRODUCTION}

La production de crevettes d'élevage est un sectcur d'activité très important pour certains pays d'Amérique du Sud et d'Extrême-Orient. Cette activité se heurte à quelques difficultés parmi lesquelles la mortalité des postlarves à l'ensemencement dans les bassins de grossissement. Cette mortalité semble liée à l'origine des animaux (écloseries, milieu naturel), aux maladies et à l'alimentation en écloseric, à la taille des postlarves ainsi qu'aux caractéristiques du nouveau 
milieu d'élevage (température, salinité, oxygène). Aussi, dans l'espoir d'une faible mortalité des postlarves, les fermiers tentent d'estimer la qualité des postlarves selon des critères visuels. Le terme qualité veut traduire l'appréciation de la condition physiologique des postlarves. Il est couramment employé par les professionnels et préjuge de la capacité des animaux de résister aux nouvelles conditions environnementales.

La résistance aux chocs de salinité est le test le plus couramment utilisé pour quantifier la condition physiologique des postlarves. Charmantier (1986) indique que "après un transfert rapide depuis l'eau de mer dans un milieu dilué, la pression osmotique de l'hémolymphe est stabilisée en 2 à $3 \mathrm{~h}$ che les postlarves P6PL4 de Penaeus japonicus". Pour cette même espèce, Charmantier et al. (1988) précisent que le temps d'adaptation de la pression osmotique de l'hémolymphe après une variation brutale dépend de la taille. S'appuyant sur ces résultats, AQUACOP (1988, donnécs non publiés) a mis au point en Équateur un test de résistance à des chocs de salinité permettant d'évaluer la qualité des postlarves de $P$. rannamei. Tackaert et al. (1989) utilisent un test similaire sur $P$. monodon sur des durées néanmoins plus courtes, dans le but de comparer la condition physiologique des postlarves dont les régimes alimentaires ont des profils en acides gras différents. Toullec et al. (1991) emploient ce test pour vérifier l'influence d'une hormone de croissance sur les postlarves de P. vannamei. Bauman et Jamandre (1990), avec P. monodon, utilisent comme stress le choc de salinité à $15 \%$ ainsi qu'un bain de formol à $100 \mathrm{mg} / \mathrm{l}$.

Cette étude a pour objectif de proposer un modèle statistique de résistance des postlarves de $P$. zannamei du $1^{\text {er }}$ au $20^{\text {e }}$ jour après la métamorphose à 5 chocs de salinité en fonction de la croissance.

\section{MATÉRIEL ET MÉTHODE}

\section{Les animaux}

Ce travail est mené à IFREMER, au Centre Océanologique du Pacifique, situé à Tahiti. Les postlarves, provenant des élcvages en cours, sont alimentées avec des nauplii vivants d'Artemia salina et des microgranulés (AQUACOP, 1990); AQUACOP et al., 1990). La température est maintenue à $29^{\circ} \mathrm{C}$ pendant les 5 premiers jours. Puis elle n'est plus contrôlée et varie entre 26 et $28^{\circ} \mathrm{C}$. La salinité est constante à $37^{\circ} \%$ o.

\section{La croissance}

Quotidiennement, 30 animaux sont mesurés vivants. On retient comme longueur totale la distance comprise entre la base du rostre et l'extrémité centrale du telson. Celte mesure est effectuée à l'aide d'une loupe binoculaire equipée d'un micromètre oculaire.
La taille des postlarves(TL) est expriméc en millimètres et l'âge en jours $(\mathrm{J})$. Une droite de régression est établie pour la croissance des postlarves entre le $2^{e}$ et $20^{e}$ jour avec 570 observations.

\section{Les chocs de salinité}

Chaque jour, 5 salinités (s) sont testées : 20, 15, 10, 5 , et $2 \%$ à la température de $27^{\circ} \mathrm{C}$. Ces milieux sont préparés dans des béchers de 11 par mélange d'cau de mer et d'eau douce. La salinité est contrôlée à l'aide d'un réfractomètre. Cinquante animaux, prélevés au hasard, sont placés dans chaque bécher sans acclimatation préalable, sans bullage ni alimentation. Le dénombrement des animaux survivants a lieu après $2 \mathrm{~h}$, temps nécessaire à la chute de la pression osmotique de l'hémolymphe (Charmanticr-Daures et al., 1988). Le critère de mortalité principal est l'absence de mouvement et en cas de doute sont considérées comme mortes les postlarves ne réagissant pas à des manipulations. Le taux de survie(S) est exprimé en pourcentage. Un modèle de régression non linéairc (modèle logistique) est proposé pour mettre en évidence le lien entre la taille moyenne journalière et le taux de survie des postlarves aux chocs de salinité.

\section{RÉSULTATS}

\section{La croissance}

L'accroissement de la taille (TL, $\mathrm{mm}$ ), entre le $2^{\mathrm{e}} \mathrm{et}$ le $20^{\mathrm{e}}$ jour $(\mathrm{J})$ est linéaire $(r=0,836 ; p<0,001)$ ( fig. 1 ).

$$
\mathrm{TL}=0,16 \mathrm{~J}+4,42
$$

\section{Les chocs de salinité}

Le test des chocs de salinité est pratiqué quotidiennement. On vérifie que la résistance des postlarves s'améliore avec l'âge donc avec la taille pour des salinités de plus en plus faibles (fig. 2). Le dixième jour, pour une salinité de $20 \%$, la survie est de $100 \%$ pour une taille moyenne de $6 \mathrm{~mm}$. On assiste à l'augmentation de la résistance pour les faibles salinités jusqu'au $20^{\circ}$ jour, où, à $2 \%, 86 \%$ des postlarves survivent.

Pour une variable évoluant non linéairement entre deux limites connues ( 0 et $100 \%$ dans notre cas), la modélisation peut s'effectuer à partir d'une fonction du type sigmoïde. L'observation du graphique du taux de survie(S) en fonction de la taille à salinité constante révèle que la forme de ces courbes est de ce type (fig. 2). Le modèle logistique sert de base pour constituer un modèle additif à deux variables explicatives (taille: TL, salinité : s). L'équation du modèle est :

$$
\mathrm{S}=100 /[1+h \cdot \exp (-c \cdot \mathrm{TL}-d . s)]
$$




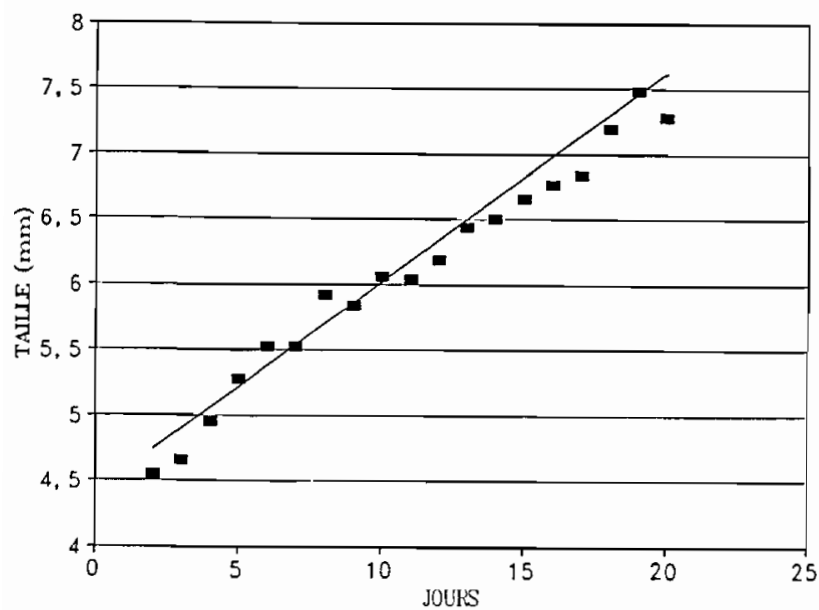

Figure 1. - Évolution de la taille des postlarves de P. vannamei pendant 20 jours.

Evolution of P. vannamei postlarvae total length during 20 days. $T L=0,16 . J+4,42\left(n=570, r^{2}=0,836, p<0,001\right)$.

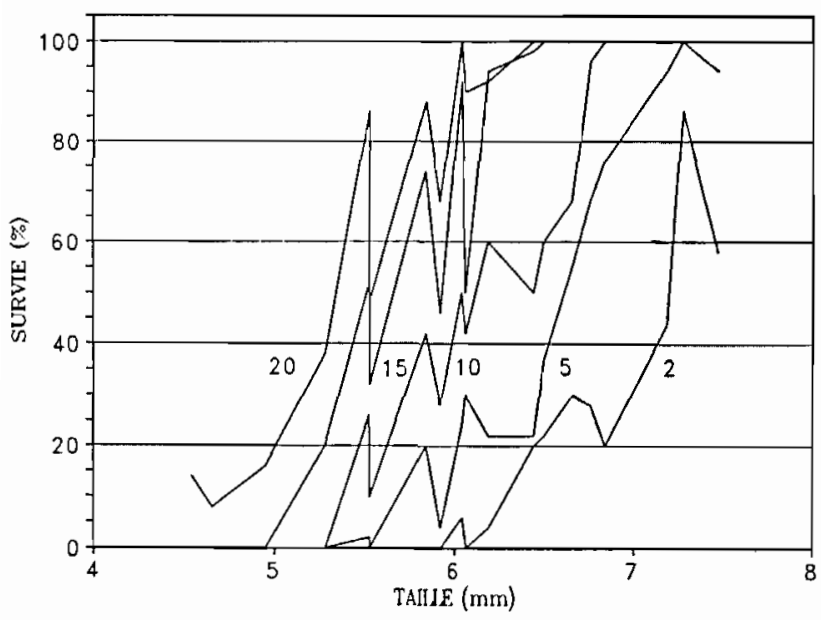

Figure 2. - Taux de survie aux chocs de salinité en fonction de la taille des postlarves $P$. vannamei (50 animaux par jour et par salinité).

Survival rate to salinity shocks in relation with $\mathrm{P}$. vannamei postlarvae total length (50 animals for each day and each salinity).

qui devient après linéarisation :

$$
\operatorname{Ln}\left(\frac{100}{\mathrm{~S}}-1\right)=\operatorname{Ln}(b)-c . \mathrm{TL}-d . s
$$

La régression effectuée sur les valeurs observées donne l'équation suivante :

$$
Z=36,545-5,158 . T L-0,440 s
$$

où

$$
\mathrm{Z}=\operatorname{Ln}[(100 / \mathrm{S})-1]
$$

$\operatorname{Ln}(b)=36,545, \quad \sigma_{\ln (b)}=1,529, c=5,158, \quad \sigma_{c}=0,243$, $d=0,440, \sigma_{d}=0,031$.

Vol. $4, n^{\circ} 3$ - 1991
Les statistiques calculées vérifient la bonne qualité du modèle. Le coefficient de détermination $r^{2}=0,876$ (test de Fisher, $p<0,001$ ) est satisfaisant (le modèle explique $87,6 \%$ de la variance totale des observations). Les coefficients de l'ćquation (4) sont tous très significativement différents de 0 (test de Student,

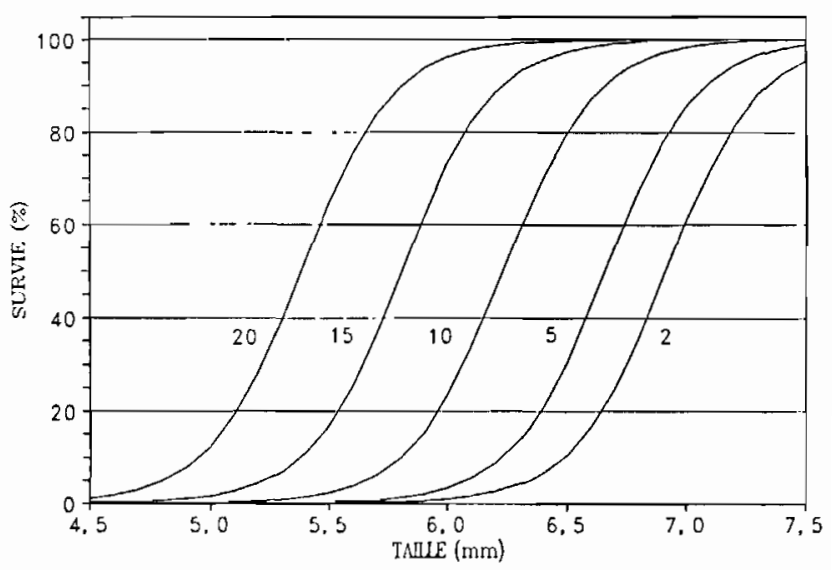

Figure 3. - Modélisation de la résistance aux chocs de salinité a $20,15,10,5$ et $2 \%$ des postlarves de P. zannamei en fonction de la taille.

Modelling of resistance to salinity shocks at 20, 15, 10, 5 and $2 \%$ of salinity of $\mathbf{P}$. vannamei postlarzat in relation with the size.

$p<0,001$ pour chacun). Le modèle estimé (fig. 3) est done :

$$
S=100 /[1+\exp (36,545)
$$

$\mathrm{S}:$ taux de survie, TL : longueur totale des postlarves $(\mathrm{mm}), s:$ salinité $(\% / 00)$.

Il faut prévoir des effectifs survivants minimum théoriques d'après le modèle (équation 6) supéricurs ou égaux à 5, cependant la correction de Yates est envisageable pour des effectifs théoriques compris entre 3 et 5 (Schwartz, 1963). Pour remplir ces conditions d'utilisation, il faut donc choisir des salinités susceptibles, pour une taille donnée, d'y répondre (tabl. 1).

\section{DISCUSSION}

La croissance des postlarves de $P$. vannamei est linéaire jusqu'au $20^{\mathrm{e}}$ jour après la métamorphose. Cette relation est importante dans la mesure où la taille est une variable explicative plus pertinente que l'âge. Le paramètre taille intègre les effets sur la croissance des variations biologiques et environnementales. La tolérance à la salinité des postlarves de $P$. vannamei augmente avec la taille pour des variations de salinité de plus en plus importantes. Ces résultats expliquent l'aptitude des postlarves de 
Tableau 1. - Chocs de salinité applicables aux classes de taillo (TL) des postlarves de $P$. vannamei.

Salinity shocks applicable to P. vannamei postlarzae at zarious size classe's $(T L)$.

\begin{tabular}{cc}
$\begin{array}{c}\text { Salinité } \\
(\% / 00)\end{array}$ & $\begin{array}{c}\text { Classes } \\
\text { de taille } \\
(\mathrm{mm})\end{array}$ \\
\hline 20 & $5,00 \leqq \mathrm{TL} \leqq 5,80$ \\
15 & $5,40 \leqq \mathrm{TL} \leqq 6,20$ \\
10 & $5,90 \leqq \mathrm{TL} \leqq 6,70$ \\
5 & $6,30 \leqq \mathrm{TL} \leqq 7,10$ \\
2 & $6,50 \leqq \mathrm{TL} \leqq 7,30$ \\
\hline
\end{tabular}

pénéides à réguler leur milieu intéricur face aux variations de leur environnement.

Les aptitudes osmorégulatrices sont différentes entre les juvéniles et les adultes chez plusieurs espèces de pénéides (Dall, 1981; Castille et Lawrence, 1981). Charmantier (1986) précise pour P. japonicus les principales caractéristiques de l'osmorégulation des larves et des postlarves qui est respectivement hyper-osmotique et hyper-hypo-osmotique. Ce dernier type de régulation est caractéristique des adultes. L'osmorégulation et la tolérance à la salinité apparaissent corrélées chez $P$. japonicus. La tolérance à la salinité augmente avec le stade de développement pendant que le temps de stabilisation de la pression osmotique de l'hémolymphe s'accroît (Charmanticr et al., 1988). Les conditions environnementales telles que la température ont un rôle prépondérant sur l'osmorégulation et la tolérance à la salinité chez les postlarves de $P$. japonicus et $P$. orientalis (Charmantier-Daures et al., 1988). La régulation de type hyper-hypoosmotique ne se développe que lorsque les structures branchiales et un certain niveau d'activité de l'ATPase $\mathrm{Na}^{+}-\mathrm{K}^{+}$existent (Bouaricha, 1990). Ce même auteur, étudiant l'ontogenèse de l'osmorégulation chez $P$. japonicus, détecte une importante activité ATPasique dans le céphalothorax essentiellement au niveau branchial, où se situe l'essentiel des échanges ioniques. Le maximum de l'activité de cette enzyme se situe à PL 5.

Le modèle de résistance aux chocs de salinité est construit à partir d'un élevage conduit selon des normes déterminées par AQUACOP (1990) et AQUACOP et al. (1990). Ce modèle permet de juger la qualité relative des postlarves avant ensemencement. Pour cela, le taux de survie théorique est calculé en fonction de la taille moyenne du lot et de la salinité testéc (équation 6). Le taux de survic réel est comparé à celui du modèle par un test de $\chi^{2}$. Le test de résistance aux chocs de salinité permet aussi de distinguer plusieurs lots de même taille moyenne. Dans ce cas, il n'est pas nécessaire de passer par le modèle, seuls les taux de survic réclle sont comparés directement à laide du $\chi^{2}$.

Les résultats du test ainsi que lc modèle mis au point ne sont pas considérés comme prédictifs des rendements ultéricurs. Il serait d'ailleurs hasardeux de le prendre comme tel dans l'état actuel de la maitrise du grossissement dans des régions comme l'Amérique du Sud. Des facteurs tels que la gestion de l'eau et la qualité des aliments limitent les rendements (Aiken, 1990). Il est certain cependant que des postlarves de moindre résistance (en deçà des limites inféricures du modèle) présentent des performances ultéricures médiocres (AQUACOP, donnćes non publiées). Le modèle de résistance aux chocs de salinité est aussi un outil permettant de mieux appréhender les cinétiques de baisse de la salinité pour l'acclimatation des postlarves de $P$. vannamei d'écloserie à des eaux de faible salinité.

\section{RÉFÉRENCES}

Aiken D., 1990. Shrimp farming in Ecuador. World Aquaculture, 21, 48-55.

AQUACOP, 1990. Produccion intensiva de postlarvas de camaron en Tahiti. Aplicacion a eclosionadores comerciales. In: La Acuacultura del Camaron, Langostino y Cangrejo en el Mundo: Bases y Technologias. Claudio Chave $z$ J. (ed.), Tokyo University of Fisheries.

AQUACOP, G. Cuzon, G. Le Moullac, 1990. El uso de dictas microparticuladas en la alimentacion larvaria del camaron. In: La Acuacultura del Camaron, Langostino y Cangrejo en el Mundo: Bases y Technologias. Claudio Chavez J. cd., Tokyo University of Fisheries.

Bauman R. H., D. R. Jamandrc, 1990. A practical method for determining quality of Penaeus monodon (Fabricius) fry for stocking in grow-out ponds. Aquatech'90 International Conference on Shrimp Culture; 11-14 Junc 1990, Kuala Lumpur, Malaysia.

Bouaricha N., 1990. Ontogenèse de l'osmorégulation chez la crevettc Penaeus japonicus. Thèse dr. Univ. Montpellier, $158 \mathrm{p}$.

Castille F. L. Jr, A. L. Lawrence, 1981. A comparison of the capabilities of juvenile and adult Penaeus setiferus and $P$. stylirostris to regulate the osmotic, sodium and chloride concentration in the hemolymph. Comp. Biochem. Physiol., 68A, 677-680.

Charmantier G., 1986. Variation des capacités osmorégulatrices au cours du développement postembryonnaire de Penaeus japonicus. C. R. Acad. Sc. Paris, 303, Sćr. III, $n^{\circ} 6$.

Charmantier G., M. Charmantier-Daures, N. Bouaricha, P. Thuet, D. E. Aiken, J.-P. Trilles, 1988. Ontogeny of osmoregulation and salinity tolerance in two decapod crustaceans: Homarus americamus and Penaeus japonicus. Biol. Bull., 175, 102-110.

Charmantier-Daures M., P. Thuet, G. Charmantier, J. P. Trilles, 1988. Tolérance à la salinité et osmoréguJation chez les postlarves de Penaeus japonicus et $P$. chinensis. Effet de la température. Aquat. Living Resour., 1, 267-276.

Dall W., 1981. Osmoregulatory ability and juvenile habitat preference in some penacid prawns. J. Exp. Mar. Biol. Ecol., 54, 55-64. 
Schwartz. D., 1963. Méthodes statistiques à l'usage des médecins et des biologistes. Flammarion Médecine Sciences, $306 \mathrm{p}$.

Tackacrt W., P. Abelin, Ph. Dhert, P. Sorgeloos, 1989. Stress resistance in postlarval penacid shrimp reared under different feeding procedures (abstract). J. World Aquac. Soc., 20, $74 \mathrm{~A}$.
Tomassone R., E. Lesquoy, C. Miller, 1983. La régression. Nouvcaux regards sur une ancienne méthode statistique. INRA. Masson, $177 \mathrm{p}$.

Toullec J. Y., G. Le Moullac, G. Cuzon, A. Van Wormhoudt, 1991. Immunoreactive human growth hormone like peptides in tropical penacids and the efrect of dietary hGH on Penaeus vannamei larval devclopment. Aquat. Lizing Resour.. 4, 125-132. 